\title{
Flat structures on the deformations of Gepner chiral rings
}

\author{
Alexander Belavin ${ }^{a, c}$ and Vladimir Belavin ${ }^{b, c, d}$ \\ ${ }^{a}$ L.D. Landau Institute for Theoretical Physics, \\ Akademika Semenova av., 1-A, Chernogolovka, 142432 Moscow region, Russia \\ ${ }^{b}$ I.E. Tamm Department of Theoretical Physics, P.N. Lebedev Physical Institute, \\ Leninsky av., 53, 119991 Moscow, Russia \\ ${ }^{c}$ Department of Quantum Physics, Institute for Information Transmission Problems, \\ Bolshoy Karetny per. 19, 127994 Moscow, Russia \\ ${ }^{f}$ Moscow Institute of Physics and Technology, \\ Dolgoprudnyi, 141700 Moscow region, Russia \\ E-mail: belavin@itp.ac.ru, belavin@lpi.ru
}

Abstract: We propose a simple method for the computation of the flat coordinates and Saito primitive forms on Frobenius manifolds of the deformations of Jacobi rings associated with isolated singularities. The method is based on using a conjecture about integral representations for the flat coordinates and on the Saito cohomology theory. This reduces the computation to a simple linear problem. We consider the case of the deformed Gepner chiral rings. The knowledge of the flat structures of Frobenius manifolds can be used for exact solution of the models of the topological conformal field theories corresponding to these chiral rings.

Keywords: 2D Gravity, Conformal and W Symmetry, Conformal Field Models in String Theory, Topological Field Theories

ARXIV EPRINT: 1606.07376 


\section{Contents}

1 Introduction 1

2 Singularities, Jacobi rings and Frobenius manifolds 3

2.1 Dubrovin axioms 4

2.2 K. Saito primitive form 5

3 Approach for the computation of the flat coordinates $\quad 6$

3.1 Integral representation for flat coordinates on FM 6

3.2 Saito cohomologies and exact expressions for Flat coordinates $\quad 7$

4 Computation of the flat coordinates for Gepner chiral rings $\quad 8$

4.1 Gepner chiral rings and their deformations 8

4.2 Computation of the flat coordinates for $\mathrm{SU}(3)_{3}$ chiral ring 9

4.3 Explicit expressions for the flat coordinates for $t_{10}=0 \quad 11$

4.4 Explicit expressions for the flat coordinates for $t_{10} \neq 0 \quad 12$

5 Conclusion 14

\section{Introduction}

In this paper we study flat structures [1] on Frobenius manifolds (FMs) [2] arising on the deformations of isolated singularities. The physical interest to the singularity theory [3] is motivated by its connection $[4,5]$ with the chiral rings of $\mathcal{N}=2$ superconformal field theories (SCFTs).

The models of $\mathcal{N}=2$ SCFT, classified and explicitly constructed in [6], are important due to their role in string theory. It was shown in [7] that $\mathcal{N}=2$ superconformal symmetry on the world sheet after compactifying six of the ten dimensions is the necessary condition for the space-time supersymmetry. The requirement of $\mathcal{N}=2$ supersymmetry in the compact sector is equivalent [8] to the geometrical condition [9] of the compactification on Calabi-Yau (CY) manifolds. The massless sector of string theory corresponds to the set of chiral fields of $\mathcal{N}=2$ SCFT and after Witten's twist [10] it is described by the topological CFT (TCFT). The properties of the Lagrangian of the sigma model describing this massless sector are completely defined in terms of the CY manifold and its mirror pair $[12,13]$.

The FM structure in TCFT was initially discovered in [14] and later mathematically developed in [2]. In [14] it was shown that the exact solution of TCFT, that is the computation of the correlation functions, is reduced to the computation of the flat coordinates on the corresponding FM. This is a specific property of $\hat{U}(2)_{k} \times \hat{U}(1)_{1} / \hat{U}(1)_{k+1}$ models 
considered in [14]. In the general case of $\mathcal{N}=2$ SCFT, associated with some isolated singularity one needs also to know the Saito primitive form [1].

It was established recently [15-23] that the knowledge of FM structure allows to find exact solution of a special class of models of non-critical string theory (or models of Minimal Liouville gravity) [24-27]. It is natural to assume that the same approach can also help to solve the $\mathcal{W}$ generalizations of Liouville gravity. The solution of the Douglas string equation [28], required for the construction of the free energy, takes simple form only in the flat coordinates on the associated FM [19]. Otherwise the integral representation of the free energy is too complicated to be of any practical use.

The explicit form of the flat coordinates is important also for the solution of the TCFT models related to the Gepner chiral rings [29]. It was shown in [29] that these models, described by the Kazama-Suzuki (KS) cosets $A(N, k)=\hat{U}(N)_{k} \times \hat{U}(N-1)_{1} / \hat{U}(N-1)_{k+1}$, are connected with a certain class of isolated singularities. The super-potentials in this case is

$$
W_{0}\left(q_{1}, \ldots, q_{N-1}\right)=\frac{1}{N+k} \sum_{i=1}^{N-1} q_{i}^{N+k} .
$$

Thus, the computation of the flat coordinates and the Saito primitive form for the Frobenius manifolds represents a significant part of the solution of the above mentioned problems.

In this paper we suggest a new simple method for computing flat coordinates on the FM arising on the deformations of the isolated singularities. This problem was discussed earlier in $[1,30-33]$. Our approach is based on the conjecture about integral representations for the flat coordinates and on the Saito cohomology theory [1]. This method allows to reduce the problem of the computation of the flat coordinates and the Saito primitive form [1] to a linear problem. Namely, we show that the coefficients of the decomposition of the flat coordinates in a sum of monomials, constructed from the deformation parameters, are simply connected with the coefficients of the decomposition of the dual to these monomials differential forms in a sum over basis elements in the space of the Saito cohomologies.

We apply this approach to the computation of the flat coordinates and the primitive form for Gepner chiral rings in the particular case of $A(3, k)=\hat{U}(3)_{k} \times \hat{U}(2)_{1} / \hat{U}(2)_{k+1}$ models. This series is interesting because, unlike previously studied $A(2, k)$ series, in this case the marginal and irrelevant deformations are present in the spectrum. We demonstrate the general construction in the particular case $k=3$ and find explicit formulae for the flat coordinates and the primitive form in terms of hypergeometric functions. The linear system for the computation of the flat coordinates together with the conjecture about the integral representation for them is the main result of the present paper.

The rest of this paper is organized as follows. In section 2 we discuss the Jacobi rings and recall some known facts about Frobenius manifolds and their relations with the Saito theory. In section 3 we develop our method for computing the flat coordinates and discuss the problem of finding the primitive form. We formulate here the conjecture about the integral representation for the flat coordinates for isolated singularity, whose Jacoby ring includes the marginal deformations in addition to the relevant ones. Further, 
we describe the linear system for the computation of the flat coordinates in terms of the deformation parameters. In section 4 we implement the proposed procedure to calculate the flat structure of the FM associated with the Gepner chiral rings and present the details of the computation for the $\mathrm{SU}(3)_{3}$ case. In section 5 we conclude by summarizing and discussing some future perspectives.

\section{Singularities, Jacobi rings and Frobenius manifolds}

Here we recall the main facts about the connection between Frobenius manifolds and topological conformal field theories relevant for our further discussion.

Dijkgraaf, Verlinde, and Verlinde (DVV) have found [14] exact solution for the minimal models of TCFT. These models are Landau-Ginzburg models whose superpotential

$$
W_{0}(x)=\frac{1}{k+2} x^{k+2} .
$$

The correlation functions in these models are defined by the free energy (also called frobenius prepotential) $\mathcal{F}\left(s^{1}, \ldots s^{k+1}\right)$, where $s^{\mu}$ are the coupling constants of the theory and $s^{1}$ corresponds to the unity operator. The perturbed three-point function

$$
C_{\alpha \beta \gamma}(s)=\left\langle\Phi_{\alpha} \Phi_{\beta} \Phi_{\gamma} \exp \sum_{\sigma} s^{\sigma} \int d^{2} z \Phi_{\sigma}^{1,1}(z)\right\rangle
$$

is given by

$$
C_{\alpha \beta \gamma}=\frac{\partial^{3} \mathcal{F}}{\partial s^{\alpha} \partial s^{\beta} \partial s^{\gamma}} .
$$

It has been shown in [14] that because of remarkable properties of TCFT all the information about arbitrary $n$-point correlation functions is encoded in the two basic objects: the threeand the two-point functions. The perturbed two-point function $\eta_{\alpha \beta}$ given explicitly by

$$
\eta_{\alpha \beta}=C_{1 \alpha \beta}=\left\langle\Phi_{\alpha} \Phi_{\beta} \exp \sum_{\sigma} s^{\sigma} \int d^{2} z \Phi_{\sigma}^{1,1}(z)\right\rangle
$$

where $\Phi_{\alpha}(z)$ is a chiral field and $\Phi_{\sigma}^{1,1}(z)$ is its superpartner.

Using CFT Ward identities it has been proven in [14] that $\eta_{\alpha \beta}$ and $C_{\alpha \beta \gamma}(s)$ should satisfy to the following set of the constraints

$$
\begin{aligned}
\partial_{\delta} C_{\alpha \beta \gamma} & =\partial_{\alpha} C_{\delta \beta \gamma}, \\
C_{\alpha \beta}^{\rho} C_{\rho \gamma}^{\delta} & =C_{\alpha \rho}^{\delta} C_{\beta \gamma}^{\rho} . \\
\partial_{\alpha} \eta_{\mu \nu} & =0, \\
C_{\mu \nu \lambda} & =C_{\nu \mu \lambda}=C_{\nu \lambda \mu} .
\end{aligned}
$$

Here, in the second relation (associativity condition), raising indices is performed by the inverse tensor $\eta^{\lambda \rho}$. From the eqs. (2.5)-(2.8) it follows that $C_{\mu \nu}^{\lambda}$ is a structure constant 
of some Frobenius algebra. ${ }^{1}$ In particular, one of the main properties of the Frobenius algebra, eq. (2.3), follows from (2.5) and (2.8). These relations mean that we are dealing with a Riemannian manifold, provided with some additional algebraic structure, which is compatible with the Riemannian one. The parameters $s^{\alpha}$ play role of the coordinates on this manifold and $\eta_{\rho \nu}$ defines its metric. We note that the third relation (2.7) states that this manifold is flat. In fact, the relations (2.5)-(2.8) define the structure of the Frobenius underlying TCFT.

\subsection{Dubrovin axioms}

The parameters $s_{\mu}$ define a special choice of flat coordinates on $M$. The metric $\eta^{\mu \nu}$ in these coordinates is constant. In some general coordinate frame $t=\left\{t^{\mu}\right\}$ the Frobenius manifold axioms look like

$$
\begin{aligned}
\nabla_{\delta} \widetilde{C}_{\alpha \beta \gamma} & =\nabla_{\alpha} \widetilde{C}_{\delta \beta \gamma}, \\
\widetilde{C}_{\alpha \beta}^{\rho} \widetilde{C}_{\rho \gamma}^{\delta} & =\widetilde{C}_{\alpha \rho}^{\delta} \widetilde{C}_{\beta \gamma}^{\rho}, \\
\widetilde{C}_{\mu \nu \lambda} & =\widetilde{C}_{\nu \mu \lambda}=\widetilde{C}_{\nu \lambda \mu}, \\
R_{\mu \nu \lambda \sigma}\left[g_{\alpha \beta}\right] & =0,
\end{aligned}
$$

where $\nabla_{\mu}$ is the covariant derivative and $R_{\mu \nu \lambda \sigma}$ is Riemann curvature tensor and the structure constants in the new coordinates are denoted by $\widetilde{C}_{\alpha \beta}^{\gamma}(t)$.

It has been proven in [14] for the case of the minimal models that the constants $C_{\mu \nu}^{\lambda}(s)$ are the structure constants in the flat coordinates of the Fobenius manifold connected with the deformed isolated singularity

$$
W=W_{0}+\sum_{\mu=1}^{k+1} t^{\mu} e_{\mu}(x),
$$

where $e_{\mu}(x)=x^{\mu-1}$ and the deformation parameters $t^{\mu}$ represent some coordinates on the FM. The parameter $t^{1}$ here and in what follows corresponds to the unity operator $e_{1}$ of the Jacobi ring.

In the $t$-frame the structure constants are defined by

$$
e_{\mu} e_{\nu}=\widetilde{C}_{\mu \nu}^{\lambda}(t) e_{\lambda} \bmod \partial W / \partial x
$$

The metric is

$$
g_{\mu \nu}=\operatorname{Res}_{x=\infty} \frac{e_{\mu} e_{\nu} \Omega}{\partial W / \partial x}
$$

where $\Omega$ is the Saito primitive form. In the case of minimal models

$$
\Omega=d x
$$

\footnotetext{
${ }^{1}$ We recall that Frobenius algebra is a commutative and associative algebra with unity such that the multiplication low is agreed in a natural way with the definition of the paring (2.8). Frobenius manifold represents a family of Frobenius algebras depending on a number of parameters. For more details see, e.g., [2].
} 
According to the statement formulated above, in order to solve the model we need to know the flat coordinates $s^{\mu}(t)$ on the FM as functions of the deformation parameters. If the metric is flat, the flat coordinates $s^{\mu}(t)$ are given by the solutions of the equation

$$
\nabla_{\alpha} \nabla_{\beta} s^{\mu}=\Gamma_{\alpha \beta}^{\gamma} \nabla_{\gamma} s^{\mu},
$$

where $\Gamma_{\alpha \beta}^{\gamma}$ is Levi-Civita connection for $g_{\alpha \beta}(t)$.

The free energy $\mathcal{F}$ is defined from

$$
C_{\mu \nu \lambda}=\frac{\partial^{3} \mathcal{F}}{\partial s^{\mu} \partial s^{\nu} \partial s^{\lambda}}
$$

and

$$
C_{\mu \nu \lambda} \frac{\partial s^{\mu}}{\partial t^{\alpha}} \frac{\partial s^{\nu}}{\partial t^{\beta}} \frac{\partial s^{\lambda}}{\partial t^{\gamma}}=\operatorname{Res}_{x=\infty} \frac{e_{\alpha} e_{\beta} e_{\gamma} \Omega}{\partial W / \partial x_{1} \cdots \partial W / \partial x_{n}} .
$$

In fact, the eqs. (2.18) and (2.19) give us the exact solution of the minimal models of TCFT as soon as we know the explicit expression for the flat coordinates as functions of the deformation parameters.

\subsection{K. Saito primitive form}

Let $W_{0}\left(x_{1}, \ldots, x_{n}\right)$ be a polynomial associated with an isolated quasihomogenous singularity with integer weights $\left[x_{i}\right]=\rho_{i}$ and $\left[W_{0}\right]=d$

$$
W_{0}\left(\Lambda^{\rho_{i}} x_{i}\right)=\Lambda^{d} W_{0}\left(x_{i}\right) .
$$

By definition, the corresponding Jacobi ring is given by

$$
R_{0}=\mathbb{C}\left[x_{1}, \ldots, x_{n}\right] /\left\{\frac{\partial W_{0}[x]}{\partial x_{i}}\right\} .
$$

In the analogous way as in the case of one variable, the versal deformation of the ring $R_{0}$ is given by

$$
W\left(x_{i}, t_{\alpha}\right)=W_{0}\left(x_{i}\right)+\sum_{\alpha=1}^{M} t^{\alpha} e_{\alpha}(x),
$$

where $e_{\alpha}, \alpha=1, \ldots, M$, represent a basis of the corresponding deformed Jacobi ring and $M$ is the Milnor number (the dimension of the Jacobi ring)

$$
R=\mathbb{C}\left[x_{1}, \ldots, x_{n}\right] /\left\{\frac{\partial W[x, t]}{\partial x_{i}}\right\} .
$$

We denote the weights of the homogeneous basis elements $\left[e_{\alpha}\right]=\sigma_{\alpha}$. In our convention $e_{1}$ is the unity in the ring. The following dimensional classification will be relevant. The elements $e_{\alpha}$ with $\sigma_{\alpha}<d, \sigma_{\alpha}=d$ and $\sigma_{\alpha}>d$ are called respectively relevant, marginal and irrelevant deformations.

The generalization of the eq. (2.14) is straightforward, while the analogue of (2.15) is

$$
g_{\mu \nu}=\operatorname{Res}_{x=\infty} \frac{e_{\mu} e_{\nu} \Omega}{\prod_{i} \partial_{i} W}
$$

where the primitive form

$$
\Omega(t, x)=\lambda\left(t^{\alpha}, x_{i}\right) d x_{1} \wedge \ldots \wedge d x_{n} .
$$

We will use the following important statement proved by M. Saito [34]. 
Theorem. If $W_{0}$ is an isolated singularity, then there exists such a $n$-form $\Omega[x]$ or, equivalently, a function $\lambda(x, t),[\lambda]=1$, that the above defined $C_{\alpha \beta}^{\gamma}$ and $g_{\alpha \beta}$ satisfy all $F M$ axioms (2.5)-(2.8).

We note that in the DVV case, $\lambda(x, t)=1$. In the general case, if there are marginal and/or irrelevant deformations, $\lambda(x, t) \neq 1$.

From this theorem it follows that there exist flat coordinates $s^{\mu}(t)=t^{\mu}+\mathcal{O}\left(t^{2}\right)$, such that $\left[s^{\mu}(t)\right]=\left[t^{\mu}\right]$. Additionally, the coordinate $s^{1}(t)$ is fixed by the condition that it represents a coupling of the unity operator, that is

$$
e_{1}=\frac{\partial W}{\partial s^{1}}
$$

As a consequence of this definition, one gets

$$
\frac{\partial s^{\mu}}{\partial t^{1}}=\delta_{\mu, 1},
$$

which follows from the simple argument

$$
e_{1}=\frac{\partial W}{\partial t^{1}}=\frac{\partial W}{\partial s^{\mu}} \frac{\partial s^{\mu}}{\partial t^{1}}=\frac{\partial s^{\mu}}{\partial t^{1}} e_{\mu} .
$$

Eq. (2.27) imposes the strong constraints on the primitive form discussed below. In fact, they allow to compute explicitly the primitive form simultaneously with the flat coordinates.

\section{Approach for the computation of the flat coordinates}

\subsection{Integral representation for flat coordinates on FM}

It was argued in [33], using the connection between the Jacobi rings of the deformed singularities and the Gauss-Manin (GM) systems, that the flat coordinates $s^{\mu}$ considered as functions of the parameters $t^{\mu}$ accept a simple integral representation. It was verified in [33] for the case of simple singularities, that is when the Jacobi ring includes only relevant deformations.

Here we conjecture that similar integral representations exist also in general case when the Jacobi ring includes the marginal deformations and probably irrelevant ones. The integration contours should be closed and chosen to belong to a basis in the homologies $H_{n}$ of $\mathbb{C}^{n} \backslash\{W=0\}$

$$
s^{\mu}\left(t^{\alpha}\right)=\sum_{m_{\alpha} \in \Sigma_{\mu}}\left(\int_{\gamma_{\mu}} \exp \left(W_{0}(x)\right) \prod_{\alpha} e_{\alpha}^{m_{\alpha}} \Omega\right) \prod_{\alpha} \frac{t_{\alpha}^{m_{a}}}{m_{\alpha} !},
$$

where non-negative integers $m_{\alpha} \in \Sigma_{\mu}$ if $^{2}$

$$
\sum_{\alpha} m_{\alpha}\left[t^{\alpha}\right]=\left[s^{\mu}\right]
$$

\footnotetext{
${ }^{2}$ For convenience reason in (3.1) and in what follows in order to avoid additional brackets we replace sometimes superscripts by subscripts $t_{\alpha} \equiv t^{\alpha}$ and $s_{\alpha} \equiv s^{\alpha}$. This operation has nothing to do with raising and lowering indices by the metric.
} 
In what follows we focus on the case where all elements $e_{\alpha}$ of the basis of the Jacobi ring are relevant or marginal. In this case $\Omega(t, x)$ is given by $(2.25), \lambda(t)$ is a function of only those parameters $t_{\alpha}$ which correspond to the marginal operators and does not depend on $x_{i}$. Therefore (3.1) can be rewritten in the following form

$$
s^{\mu}\left(t^{\alpha}\right)=\lambda(t) \sum_{m_{\alpha} \in \Sigma_{\mu}}\left(\int_{\gamma_{\mu}} \exp \left(W_{0}(x)\right) \prod_{\alpha} e_{\alpha}^{m_{\alpha}} d x\right) \prod_{\alpha} \frac{t_{\alpha}^{m_{\alpha}}}{m_{\alpha} !} .
$$

On the other hand, from the dimensional arguments it is obvious that in general form

$$
s^{\mu}\left(t^{1}, \ldots, t^{M}\right)=t^{\mu}+\sum_{m_{\alpha} \in \Sigma_{\mu}} C_{\mu}(\vec{m}) \prod_{\alpha=1}^{M} \frac{t_{\alpha}^{m_{\alpha}}}{m_{\alpha} !} .
$$

In what follows we show how to compute the coefficients $C_{\mu}(\vec{m})$.

\subsection{Saito cohomologies and exact expressions for Flat coordinates}

For explicit computations of the integral in (3.1) we will use the following two facts.

Firstly, two following integrals are equal

$$
\int_{\gamma} \exp \left(W_{0}(x)\right) P_{1}(x) d x=\int_{\gamma} \exp \left(W_{0}(x)\right) P_{2}(x) d x,
$$

if

$$
P_{1}(x) d x=P_{2}(x) d x+D_{W_{0}} U,
$$

where the K. Saito differential ${ }^{3}$

$$
D_{W_{0}}=d+d W_{0}
$$

and $U$ is some $(n-1)$-form

$$
U=\sum_{i=1}^{n}(-1)^{i-1} A_{i} d x_{1} \wedge \ldots \wedge d x_{i-1} \wedge d x_{i+1} \wedge \ldots \wedge d x_{n}
$$

and contour $\gamma$ is closed.

Secondly, the Saito differential defines cohomologies on the space of the differential forms. The dimension $H^{n}$ - Saito cohomology group of the highest degree is equal $M$, i.e., the dimension of the Jacoby ring of the isolated singularity $W_{0}$. As a basis $H^{n}$ can be choosen the $n$-forms $e_{\mu} d x_{1} \wedge \ldots \wedge d x_{n}$ or $e_{\mu} d x$ in short, where $e_{\mu}$ is a basis of the Jacobi ring. Since the forms $P(x) d x_{1} \wedge \ldots \wedge d x_{n}$ belong to $H^{n}$ they can be decomposed in this basis

$$
P(x) d x=\sum_{\mu} C_{\mu} e_{\mu} d x+D_{W_{0}} U .
$$

Such an expansion exists and is unique. We will use this fact to find the coefficients $C_{\mu}(\vec{m})$ from the equation

$$
\prod_{\alpha} e_{\alpha}^{m_{\alpha}} d x=\sum_{\mu} C_{\mu}(\vec{m}) e_{\mu} d x+D_{W_{0}} U
$$

\footnotetext{
${ }^{3}$ Here $d$ is the ordinary de Rham differential. We note that $D_{W_{0}}^{2}=0$.
} 
This equation can be written more explicitly as

$$
\prod_{\alpha} e_{\alpha}^{m_{\alpha}}=\sum_{\mu} C_{\mu}(\vec{m}) e_{\mu}+\sum_{i=1}^{n} A_{i}(x) \frac{\partial W_{0}}{\partial x^{i}}+\sum_{i=1}^{n} \frac{\partial A_{i}(x)}{\partial x^{i}} .
$$

Assuming that the basis contours $\gamma_{\mu}$ are chosen to be dual with elements $e_{\mu} d x$ in the basis of the cohomology space, i.e.,

$$
\int_{\gamma_{\mu}} e_{\nu} d x=\delta_{\mu, \nu}
$$

and substituting the r.h.s. in (3.10) into the integrals in (3.1) we arrive to explicit expression for the flat coordinates

$$
s^{\mu}\left(t^{\alpha}\right)=\sum_{m_{\alpha}} C_{\mu}\left(m_{\alpha}\right) \prod_{\alpha} \frac{t_{\alpha}^{m_{\alpha}}}{\left(m_{\alpha}\right) !},
$$

where the coefficients $C_{\mu}(\vec{m})$ are unique solution of the system of linear equations (3.10).

If the isolated singularity is homogeneous, the $(n-1)$-form $U$ can be represented as a sum of homogeneous terms $\sum_{l=0}^{L} U_{l}$ and the equation (3.10) takes the form

$$
\begin{aligned}
d W_{0} U_{0} & =\prod_{\lambda} e_{\lambda}^{m_{\lambda}} d x, \\
d W_{0} U_{l} & =-d U_{l-1}, \\
\sum_{\mu} C_{\mu}(\vec{m} \mid n) e_{\mu} d x & =-d U_{L},
\end{aligned}
$$

where $l=1, \ldots, L$ and $L=\sum m_{\alpha}-1$.

In the next section we will demonstrate how this procedure works in the case of the Gepner rings.

\section{Computation of the flat coordinates for Gepner chiral rings}

\subsection{Gepner chiral rings and their deformations}

Chiral rings considered by Gepner are related to the KS cosets $\hat{U}(N)_{k} \times \hat{U}(N-1)_{1} / \hat{U}(N-$ $1)_{k+1}$. Important property of these rings is that their deformations possess a structure of Frobenius manifolds. It follows [29] from the fact that these chiral rings are connected with the isolated homogeneous singularity

$$
W_{0}(x)=\frac{1}{N+k} \sum_{i=1}^{N-1} q_{i}^{N+k},
$$

and are isomorphic to the Jacoby rings

$$
R_{0}(N, k)=\mathbb{C}\left[x_{1}, \ldots, x_{N-1}\right] /\left\{\partial_{1} W_{0}[x] \ldots \partial_{N-1} W_{0}[x]\right\},
$$

where $x_{1}, \ldots, x_{N-1}$ are elementary symmetric polynomials of $q_{1}, \ldots, q_{N-1}$ :

$$
x_{1}=\sum_{i=1}^{N-1} q_{i}, \quad x_{2}=\sum_{i<j}^{N-1} q_{i} q_{j}, \quad \ldots, \quad x_{N-1}=q_{1} \ldots q_{N-1} .
$$


Note that the dimension of the ring is

$$
\operatorname{dim} R_{0}(N, k)=\frac{(N+k-1) !}{k !(N-1) !} .
$$

Taking into account symmetric property of the ring space it is clear that Schur polynomials

$$
S_{\lambda}\left[q_{1}, \ldots, q_{N-1}\right]=\frac{\operatorname{det} q_{i}^{N+\lambda_{j}-j}}{\operatorname{det} q_{i}^{N-j}}
$$

can be chosen as a natural homogeneous basis in $R^{0}(N, k)$. We remind that $\lambda$ in (4.5) stands for the Young diagram $\lambda=\left(\lambda_{1}, \ldots, \lambda_{N-1}\right)$, with $\lambda_{1} \geq \lambda_{2} \geq \ldots \geq \lambda_{N-1} \geq 0$ and $0 \leq \lambda_{1} \leq k$.

\subsection{Computation of the flat coordinates for $\mathrm{SU}(3)_{3}$ chiral ring}

For $k \geq 3$ the consideration of the models associated with the Gepner chiral rings (4.2) become more involved because marginal deformations appear in the spectrum. Let us consider the particular case $k=3$ where

$$
W_{0}\left(x_{1}, x_{2}\right)=\frac{1}{6}\left(q_{1}^{6}+q_{2}^{6}\right) .
$$

According to (4.4) the dimension of the ring space $\operatorname{dim} R_{0}(3,3)=10$. The basis elements are enumerated by Young diagrams restricted to a rectangle of size $k \times(N-1)$ with $k=3$, $N=3$. For convenience we will use the following identification

$$
\begin{aligned}
& e_{1} \equiv e_{\varnothing}=1, \\
& e_{2} \equiv e_{\text {口 }}=q_{1}+q_{2}, \\
& e_{3} \equiv e_{\text {日 }}=q_{1} q_{2}, \\
& e_{4} \equiv e_{\text {甲 }}=q_{1}^{2}+q_{1} q_{2}+q_{2}^{2}, \\
& e_{5} \equiv e_{\text {甲 }}=q_{1} q_{2}\left(q_{1}+q_{2}\right), \\
& e_{6} \equiv e_{\text {四 }}=q_{1}^{3}+q_{1}^{2} q_{2}+q_{1} q_{2}^{2}+q_{2}^{3}, \\
& e_{7} \equiv e_{\boxplus}=q_{1}^{2} q_{2}^{2}, \\
& e_{8} \equiv e_{\text {田 }}=q_{1} q_{2}\left(q_{1}^{2}+q_{1} q_{2}+q_{2}^{2}\right), \\
& e_{9} \equiv e_{\boxplus}=q_{1}^{2} q_{2}^{2}\left(q_{1}+q_{2}\right), \\
& e_{10} \equiv e_{\boxplus}=q_{1}^{3} q_{2}^{3} .
\end{aligned}
$$

After the versal deformation the potential reads

$$
W\left(q_{1}, q_{2}, t\right)=\frac{q_{1}^{6}+q_{2}^{6}}{6}+\sum_{l=1}^{10} t^{l} e_{l} .
$$

Note that in this particular case there is only one marginal deformation which is presented by $e_{10}$, all other perturbations $e_{1}, \ldots, e_{9}$ in (4.8) are relevant. Therefore we have to allow 
the primitive form (2.25) to be a function of the dimensionless coupling $t_{10}$. The coordinates in the constant metric coordinate system are given by the following general expression

$$
s^{\mu}\left(t^{\alpha}\right)=\lambda\left(t_{10}\right) \sum_{\left\{m_{\lambda}\right\} \in \Sigma_{\mu}} \sum_{n=0}^{\infty} C_{\mu}\left(m_{\lambda} \mid n\right) \prod_{1 \leq \lambda<10} \frac{t_{\lambda}^{m_{\lambda}}}{m_{\lambda} !} \frac{t_{10}^{n}}{n !},
$$

where $\Sigma_{\mu}$ is defined in (3.2). In accordance with our main conjecture (3.1) the coefficients $C_{\mu}\left(m_{\lambda} \mid n\right)$ are fixed by the following condition

$$
\prod_{\lambda} e_{\lambda}^{m_{\lambda}} e_{10}^{n} \Omega_{0}=\sum_{\mu} C_{\mu}\left(m_{\lambda} \mid n\right) e_{\mu} \Omega_{0}+D_{W_{0}} U
$$

where

$$
\Omega_{0}=\left(q_{1}-q_{2}\right) d q_{1} \wedge d q_{2},
$$

and the sum in (4.10) is over those $\mu$, which are compatible with the dimensional restriction (3.2) for given set of $m_{\lambda}$. The 1-form $U$ can be conveniently represented as

$$
U=A\left(q_{1}, q_{2}\right) d q_{1}+B\left(q_{1}, q_{2}\right) d q_{2},
$$

such that $B\left(q_{1}, q_{2}\right)=A\left(q_{2}, q_{1}\right)$. More explicitly we find

$$
\prod_{\lambda} e_{\lambda}^{m_{\lambda}} e_{10}^{n}\left(q_{1}-q_{2}\right)=\sum_{\mu} C_{\mu}\left(m_{\lambda} \mid n\right) e_{\mu}\left(q_{1}-q_{2}\right)+\frac{\partial B}{\partial q_{1}}-\frac{\partial A}{\partial q_{2}}+A q_{2}^{5}-B q_{1}^{5} .
$$

Taking into account the argumentation of section 3, we can transform this relation into the set of recurrence relations for its decomposition into homogeneous pieces

$$
A=\sum_{s=0}^{L} A_{s}, \quad B=\sum_{s=0}^{L} B_{s},
$$

where $L$ is defined from the condition

$$
\left[\prod_{\lambda} e_{\lambda}^{m_{\lambda}} e_{10}^{n}\right]-\left[e_{\mu}\right]=6 L
$$

The first relation of the recurrence prescription (3.14)-(3.16) reads

$$
\prod_{\lambda} e_{\lambda}^{m_{\lambda}}\left(q_{1}-q_{2}\right)=A_{0} q_{2}^{5}-B_{0} q_{1}^{5}
$$

The second one gives

$$
\frac{\partial A_{s-1}}{\partial q_{2}}-\frac{\partial B_{s-1}}{\partial q_{1}}=A_{s} q_{2}^{5}-B_{s} q_{1}^{5}, \quad 1 \leq s \leq L .
$$

Finally, from the last one we find

$$
\sum_{\mu} C_{\mu}\left(m_{\lambda} \mid n\right) e_{\mu}\left(q_{1}, q_{2}\right)=\frac{\partial A_{L}}{\partial q_{2}}-\frac{\partial B_{L}}{\partial q_{1}} .
$$

These iterative relations allow to find all the coefficient $A_{s}, B_{s}$ and to express finally the desired $C_{\mu}\left(m_{\lambda} \mid n\right)$ in terms of the highest coefficients $A_{L}, B_{L}$. 


\subsection{Explicit expressions for the flat coordinates for $t_{10}=0$}

Now we want to find the flat coordinates. Our first step is to consider the situation with the marginal perturbation switched off. In this case the most general coordinate transformation which is compatible with the requirement of quasihomogeneity condition (2.20) can involve only the following contributions

$$
\begin{aligned}
s_{10}: & \left\{t_{10}\right\} \\
s_{9} & :\left\{t_{9}\right\} \\
s_{8} & :\left\{t_{8}, t_{9}^{2}\right\} \\
s_{7}: & \left\{t_{7}, t_{9}^{2}\right\} \\
s_{6}: & \left.: t_{6}, t_{8} t_{9}, t_{7} t_{9}, t_{9}^{3}\right\} \\
s_{5}: & \left\{t_{5}, t_{8} t_{9}, t_{7} t_{9}, t_{9}^{3}\right\} \\
s_{4}: & \left\{t_{4}, t_{6} t_{9}, t_{5} t_{9}, t_{8} t_{9}^{2}, t_{7} t_{9}^{2}, t_{8}^{2}, t_{7}^{2}, t_{7} t_{8}, t_{9}^{4}\right\} \\
s_{3}: & \left\{t_{3}, t_{6} t_{9}, t_{5} t_{9}, t_{8} t_{9}^{2}, t_{7} t_{9}^{2}, t_{8}^{2}, t_{7}^{2}, t_{7} t_{8}, t_{9}^{4}\right\} \\
s_{2}: & \left\{t_{2}, t_{4} t_{9}, t_{7}^{2} t_{9}, t_{7} t_{8} t_{9}, t_{8} t_{9}^{3}, t_{7} t_{9}^{3}, t_{9}^{5}, t_{3} t_{9}, t_{6} t_{8}, t_{6} t_{7}, t_{5} t_{8}, t_{5} t_{7}, t_{6} t_{9}^{2}, t_{5} t_{9}^{2}, t_{8}^{2} t_{9}\right\}, \\
s_{1}: & :\left\{t_{1}, t_{2} t_{9}, t_{5} t_{6}, t_{6} t_{8} t_{9}, t_{6} t_{7} t_{9}, t_{5} t_{8} t_{9}, t_{5} t_{7} t_{9}, t_{6} t_{9}^{3}, t_{5} t_{9}^{3}, t_{8}^{3}, t_{7}^{3}, t_{7} t_{8}^{2}, t_{4} t_{8}, t_{7}^{2} t_{8}, t_{8}^{2} t_{9}^{2},\right. \\
& \left.t_{7}^{2} t_{9}^{2}, t_{7} t_{8} t_{9}^{2}, t_{8} t_{9}^{4}, t_{7} t_{9}^{4}, t_{9}^{6}, t_{4} t_{7}, t_{3} t_{8}, t_{3} t_{7}, t_{4} t_{9}^{2}, t_{3} t_{9}^{2}, t_{6}^{2}, t_{5}^{2}\right\} .
\end{aligned}
$$

Here each monomial listed in the right hand side comes in the expressions of the corresponding flat coordinate with a coefficient depending on $t_{10}$. For $t_{10}=0$, our approach described in the previous section gives the following explicit results

$$
\begin{aligned}
s_{10}= & t_{10}, \\
s_{9}= & t_{9} \\
s_{8}= & t_{8}+t_{9}^{2}, \\
s_{7}= & t_{7} \\
s_{6}= & t_{6}+t_{8} t_{9}+t_{7} t_{9}+\frac{5}{6} t_{9}^{3}, \\
s_{5}= & t_{5}+2 t_{8} t_{9}+\frac{8}{3} t_{9}^{3}, \\
s_{4}= & t_{4}+t_{6} t_{9}+t_{5} t_{9}+\frac{1}{2} t_{8}^{2}+t_{7} t_{8}+2 t_{7} t_{9}^{2}+4 t_{8} t_{9}^{2}+\frac{7}{2} t_{9}^{4}, \\
s_{3}= & t_{3}+2 t_{6} t_{9}+3 t_{8} t_{9}^{2}+3 t_{7} t_{9}^{2}+t_{8}^{2}+2 t_{9}^{4}, \\
s_{2}= & t_{2}+\frac{63 t_{9}^{5}}{8}+\frac{7}{2} t_{7} t_{9}^{3}+\frac{21}{2} t_{8} t_{9}^{3}+3 t_{5} t_{9}^{2}+\frac{3}{2} t_{6} t_{9}^{2}+\frac{3}{2} t_{7}^{2} t_{9}+ \\
& +3 t_{8}^{2} t_{9}+t_{4} t_{9}+3 t_{7} t_{8} t_{9}+t_{6} t_{7}+t_{5} t_{8}+t_{6} t_{8}, \\
s_{1}= & t_{1}+\frac{119 t_{9}^{6}}{15}+\frac{28}{3} t_{7} t_{9}^{4}+14 t_{8} t_{9}^{4}+\frac{7}{3} t_{5} t_{9}^{3}+\frac{13}{3} t_{6} t_{9}^{3}+\frac{13}{2} t_{8}^{2} t_{9}^{2}+t_{3} t_{9}^{2}+t_{4} t_{9}^{2}+ \\
& +2 t_{5} t_{7} t_{9}+7 t_{7} t_{8} t_{9}^{2}+2 t_{5} t_{8} t_{9}+2 t_{6} t_{8} t_{9}+\frac{t_{7}^{3}}{3}+\frac{t_{8}^{3}}{3}+t_{7} t_{8}^{2}+t_{5} t_{6}+t_{4} t_{8}-\frac{t_{6}^{2}}{2} .
\end{aligned}
$$




\subsection{Explicit expressions for the flat coordinates for $t_{10} \neq 0$}

When $t_{10} \neq 0$ the flat coordinates are series of $t_{10}$. Let us demonstrate the general idea of the previous section on the following examples for $k=3$.

In our first example we compute the contribution to $s^{1}$ which contains $t^{1}$. It has the following form

$$
s^{1}=\lambda\left(t_{10}\right) f_{1}\left(t_{10}\right) t^{1}+\ldots,
$$

where dots stand for possible contributions of other $t^{\nu}$ with $\nu \neq 1$. Our goal now is to find $f_{1}\left(t_{10}\right)$.

$$
f_{1}\left(t_{10}\right)=\sum_{n} C(n) \frac{t_{10}^{2 n}}{(2 n) !} .
$$

As explained above we compute the coefficients $C(n)$ from the equation

$$
e_{1} e_{10}^{2 n} \Omega=C(n) \Omega+D_{W_{0}} U
$$

Since $e_{1}=1$, we have to solve

$$
e_{10}^{2 n}\left(q_{1}-q_{2}\right)=C(n)\left(q_{1}-q_{2}\right)+\frac{\partial B}{\partial q_{1}}-\frac{\partial A}{\partial q_{2}}+A q_{2}^{5}-B q_{1}^{5} .
$$

It is convenient to derive the following recurrence relation for the coefficients

$$
C(n+1)=P(n) C(n) .
$$

Since $e_{10}=q_{1}^{3} q_{2}^{3}$, we get

$$
q_{1}^{6 n+6} q_{2}^{6 n+6}\left(q_{1}-q_{2}\right)=q_{1}^{6 n} q_{2}^{6 n}\left(q_{1}-q_{2}\right)+\ldots,
$$

which is to be presented in the form

$$
q_{1}^{6 n+7} q_{2}^{6 n+6}-q_{1}^{6 n+6} q_{2}^{6 n+7}=A q_{2}^{5}-B q_{1}^{5}+\frac{\partial B}{\partial q_{1}}-\frac{\partial A}{\partial q_{2}}+P(n)\left[q_{1}^{6 n+1} q_{2}^{6 n}-q_{1}^{6 n+6} q_{2}^{6 n+1}\right] .
$$

The coefficients are easily found $A_{0}=-q_{1}^{6 n+6} q_{2}^{6 n+2}, B_{0}=-q_{1}^{6 n+2} q_{2}^{6 n+6}$. So that

$$
\frac{\partial A_{0}}{\partial q_{2}}-\frac{\partial B_{0}}{\partial q_{1}}=(6 n+2)\left[q_{1}^{6 n+1} q_{2}^{6 n+6}-q_{1}^{6 n+6} q_{2}^{6 n+1}\right]
$$

which in turn is to be equal to

$$
(6 n+2)\left[q_{1}^{6 n+1} q_{2}^{6 n+6}-q_{1}^{6 n+6} q_{2}^{6 n+1}\right]=A_{1} q_{2}^{5}-B_{1} q_{1}^{5},
$$

so that $A_{1}=(6 n+2) q_{1}^{6 n+1} q_{2}^{6 n+1}, B_{1}=(6 n+2) q_{1}^{6 n+1} q_{2}^{6 n+1}$. The final condition is

$$
\frac{\partial A_{1}}{\partial q_{2}}-\frac{\partial B_{1}}{\partial q_{1}}=(6 n+1)(6 n+2)\left[q_{1}^{6 n+1} q_{2}^{6 n}-q_{1}^{6 n} q_{2}^{6 n+1}\right]=P(n)\left[q_{1}^{6 n+1} q_{2}^{6 n}-q_{1}^{6 n} q_{2}^{6 n+1}\right] .
$$

Hence,

$$
P(n)=(6 n+1)(6 n+2),
$$


and

$$
C(n)=\prod_{m=0}^{n-1}(6 m+1)(6 m+2)
$$

We conclude that

$$
f_{1}\left(t_{10}\right)=1+\sum_{n=1}^{\infty} \prod_{m=0}^{n-1}(6 m+1)(6 m+2) \frac{t_{10}^{2 n}}{(2 n) !}={ }_{2} F_{1}\left(\frac{1}{6}, \frac{1}{3} ; \frac{1}{2} \mid 9 t_{10}^{2}\right) .
$$

Taking into account the (2.27) we obtain

$$
\lambda\left(t_{10}\right)=\frac{1}{f_{1}\left(t_{10}\right)}=\frac{1}{{ }_{2} F_{1}\left(\frac{1}{6}, \frac{1}{3} ; \frac{1}{2} \mid 9 t_{10}^{2}\right)} .
$$

Our second example demonstrates the computation of the flat coordinate

$$
s_{10}=\lambda\left(t_{10}\right) t_{10} f_{10}\left(t_{10}\right)=\lambda\left(t_{10}\right) \sum_{n=1}^{\infty} C(n) \frac{t_{10}^{2 n+1}}{(2 n+1) !},
$$

with $C(0)=1$. We have the following equations for the coefficients $C(n)$

$$
e_{10}^{2 n+1} \Omega_{0}=C(n) e_{10} \Omega+D_{W_{0}} U
$$

and

$$
C(n+1)=P(n) C(n) .
$$

Or explicitly,

$$
e_{10}^{2 n+3}\left(q_{1}-q_{2}\right)=P(n) e_{10}^{2 n+1}\left(q_{1}-q_{2}\right)+A q_{2}^{5}-B q_{1}^{5}+\frac{\partial B}{\partial q_{1}}-\frac{\partial A}{\partial q_{2}} .
$$

It follows that

$$
U=U_{0}+U_{1}, \quad A=A_{0}+A_{1}, \quad B=B_{0}+B_{1},
$$

where

$$
\begin{array}{ll}
A_{0}=-q_{1}^{6 n+9} q_{2}^{6 n+4}, & B_{0}=-q_{1}^{6 n+4} q_{2}^{6 n+9} \\
A_{1}=(6 n+4) q_{1}^{6 n+3} q_{2}^{6 n+5}, & B_{1}=(6 n+4) q_{1}^{6 n+5} q_{2}^{6 n+3}
\end{array}
$$

and

$$
P(n)=(6 n+4)(6 n+5) .
$$

We find

$$
f_{10}\left(t_{10}\right)=1+\sum_{n=1}^{\infty} \prod_{m=0}^{n-1}(6 m+4)(6 m+5) \frac{t_{10}^{2 n+1}}{(2 n+1) !}
$$


One can easily see that

$$
s_{10}\left(t_{10}\right)=t_{10} \lambda\left(t_{10}\right)_{2} F_{1}\left(\frac{2}{3}, \frac{5}{6} ; \frac{3}{2} \mid 9 t_{10}^{2}\right)=t_{10} \frac{{ }_{2} F_{1}\left(\frac{2}{3}, \frac{5}{6} ; \frac{3}{2} \mid 9 t_{10}^{2}\right)}{{ }_{2} F_{1}\left(\frac{1}{6}, \frac{1}{3} ; \frac{1}{2} \mid 9 t_{10}^{2}\right)} .
$$

Let us give another few examples of the explicit results for the flat coordinates when the final expressions are not too cumbersome

$$
\begin{aligned}
& s_{9}(t)=t_{9} \frac{{ }_{2} F_{1}\left(\frac{5}{6}, \frac{1}{2} ; \frac{1}{2} \mid 9 t_{10}^{2}\right)}{{ }_{2} F_{1}\left(\frac{1}{6}, \frac{1}{3} ; \frac{1}{2} \mid 9 t_{10}^{2}\right)}, \\
& s_{8}(t)=t_{8} \frac{{ }_{2} F_{1}\left(\frac{2}{3}, \frac{1}{2} ; \frac{1}{2} \mid 3 t_{10}\right)}{{ }_{2} F_{1}\left(\frac{1}{6}, \frac{1}{3} ; \frac{1}{2} \mid 9 t_{10}^{2}\right)}+t_{9}^{2} \frac{{ }_{2} F_{1}\left(\frac{5}{3}, \frac{1}{2} ; \frac{1}{2} \mid 3 t_{10}\right)}{{ }_{2} F_{1}\left(\frac{1}{6}, \frac{1}{3} ; \frac{1}{2} \mid 9 t_{10}^{2}\right)}, \\
& s_{7}(t)=t_{7} \frac{{ }_{2} F_{1}\left(\frac{2}{3}, \frac{1}{2} ; \frac{1}{2} \mid 9 t_{10}^{2}\right)}{{ }_{2} F_{1}\left(\frac{1}{6}, \frac{1}{3} ; \frac{1}{2} \mid 9 t_{10}^{2}\right)}+6 t_{9}^{2} t_{10} \frac{{ }_{2} F_{1}\left(\frac{5}{3}, \frac{1}{2} ; \frac{1}{2} \mid 9 t_{10}^{2}\right)}{{ }_{2} F_{1}\left(\frac{1}{6}, \frac{1}{3} ; \frac{1}{2} \mid 9 t_{10}^{2}\right)} .
\end{aligned}
$$

The demonstrated above procedure, which allows to find explicit expressions for the flat coordinates, the formula for the Saito primitive form $\Omega$

$$
\Omega=\lambda\left(t_{10}\right) d x=\frac{d x}{{ }_{2} F_{1}\left(\frac{1}{6}, \frac{1}{3} ; \frac{1}{2} \mid 9 t_{10}^{2}\right)},
$$

together with the statement about the form of the flat coordinates, (3.1), (3.10), are the main results of this paper.

\section{Conclusion}

We have studied $\mathcal{N}=2 \mathrm{CFT}$ models, represented by cosets $\hat{U}(N)_{k} \times \hat{U}(N-1)_{1} / \hat{U}(N-1)_{k+1}$ for $N>2$. An important feature of these models is the presence of marginal and irrelevant perturbations. The proposed method allows to systematically analyse such situations. It gives the recurrence formula for the flat coordinates and the method to construct the primitive form. This result gives the complete solution of the model under consideration.

Among the open questions we would like to mention the following. Since the mathematical proof of the main conjecture is lacking, the direct computation of the flat coordinates, in the spirit of [35], is desirable. In the case where only relevant and marginal perturbations are presented, the check was performed in [38]. It is important to investigate the case of irrelevant perturbations.

Our results can be used in order to define the generating function of the physical amplitudes in the minimal models of $2 \mathrm{~d} \mathcal{W}$ gravity. Note that the generalization of the discrete formulation of the matrix models possessing $\mathcal{W}$ symmetry is not available at the moment. It is also worth noting that the analogue of the continuous approach, based on the explicit construction of the correlators of the chiral fields, is also absent. ${ }^{4}$

\footnotetext{
${ }^{4}$ See [39] for some development in this direction.
} 
Finally, maybe the most interesting is the possibility to investigate a new class of Calabi-Yau geometries, using the approach discussed in this paper.

\section{Acknowledgments}

We thank M. Bershtein, D. Gepner, B. Feigin, Ya. Kononov, L. Spodyneiko and G. Tarnopolsky for useful discussions. The work was performed with the financial support of the Russian Science Foundation (Grant No.14-12-01383).

Open Access. This article is distributed under the terms of the Creative Commons Attribution License (CC-BY 4.0), which permits any use, distribution and reproduction in any medium, provided the original author(s) and source are credited.

\section{References}

[1] K. Saito, Period mapping associated to a primitive form, PubL RIMS, Kyoto Univ. 19 (1983) 1231.

[2] B. Dubrovin, Integrable systems in topological field theory, Nucl. Phys. B 379 (1992) 627 [INSPIRE].

[3] V. Arnold, S. Gusein-Zade, A. Varchenko, Singularities of Differentiable Maps, Volume II: Monodromy and Asymptotic Integrals, Birkhäuser (1988).

[4] W. Lerche, C. Vafa, N. Warner, Chiral rings in $N=2$ superconformal theories, Nucl. Phys. B 234 (1989) 427.

[5] E.J. Martinec, Algebraic Geometry and Effective Lagrangians, Phys. Lett. B 217 (1989) 431 [INSPIRE].

[6] Y. Kazama and H. Suzuki, New $N=2$ Superconformal Field Theories and Superstring Compactification, Nucl. Phys. B 321 (1989) 232 [INSPIRE].

[7] D. Gepner, Space-Time Supersymmetry in Compactified String Theory and Superconformal Models, Nucl. Phys. B 296 (1988) 757 [inSPIRE].

[8] D. Gepner, Scalar Field Theory and String Compactification, Nucl. Phys. B 322 (1989) 65 [INSPIRE].

[9] P. Candelas, G.T. Horowitz, A. Strominger and E. Witten, Vacuum Configurations for Superstrings, Nucl. Phys. B 258 (1985) 46 [InSPIRE].

[10] E. Witten, Topological Quantum Field Theory, Commun. Math. Phys. 117 (1988) 353 [INSPIRE].

[11] T. Eguchi and S.-K. Yang, $N=2$ superconformal models as topological field theories, Mod. Phys. Lett. A 5 (1990) 1693 [inSPIRE].

[12] P. Candelas, X.C. De La Ossa, P.S. Green and L. Parkes, A pair of Calabi-Yau manifolds as an exactly soluble superconformal theory, Nucl. Phys. B 359 (1991) 21 [INSPIRE].

[13] P. Berglund et al., Periods for Calabi-Yau and Landau-Ginzburg vacua, Nucl. Phys. B 419 (1994) 352 [hep-th/9308005] [INSPIRE].

[14] R. Dijkgraaf, H.L. Verlinde and E.P. Verlinde, Topological strings in $d<1$, Nucl. Phys. B 352 (1991) 59 [INSPIRE]. 
[15] A.A. Belavin and A.B. Zamolodchikov, On Correlation Numbers in 2D Minimal Gravity and Matrix Models, J. Phys. A 42 (2009) 304004 [arXiv:0811.0450] [INSPIRE].

[16] A. Belavin and G. Tarnopolsky, Two dimensional gravity in genus one in Matrix Models, Topological and Liouville approaches, JETP Lett. 92 (2010) 257 [arXiv:1006.2056] [INSPIRE].

[17] G. Tarnopolsky, Five-point Correlation Numbers in One-Matrix Model, J. Phys. A 44 (2011) 325401 [arXiv: 0912 .4971] [INSPIRE].

[18] A. Belavin, B. Dubrovin and B. Mukhametzhanov, Minimal Liouville Gravity correlation numbers from Douglas string equation, JHEP 01 (2014) 156 [arXiv:1310.5659] [INSPIRE].

[19] V. Belavin, Unitary Minimal Liouville Gravity and Frobenius Manifolds, JHEP 07 (2014) 129 [arXiv: 1405.4468] [INSPIRE].

[20] A.A. Belavin and V.A. Belavin, Frobenius manifolds, Integrable Hierarchies and Minimal Liouville Gravity, JHEP 09 (2014) 151 [arXiv:1406.6661] [INSPIRE].

[21] L. Spodyneiko, Minimal Liouville Gravity on the Torus via Matrix Models, arXiv: 1407.3546 [INSPIRE].

[22] V. Belavin and Yu. Rud, Matrix model approach to minimal Liouville gravity revisited, J. Phys. A 48 (2015) 18FT01 [arXiv:1502.05575] [inSPIRE].

[23] V. Belavin, Correlation Functions in Unitary Minimal Liouville Gravity and Frobenius Manifolds, JHEP 02 (2015) 052 [arXiv: 1412.4245] [INSPIRE].

[24] A.M. Polyakov, Quantum Geometry of Bosonic Strings, Phys. Lett. B 103 (1981) 207 [INSPIRE].

[25] V.G. Knizhnik, A.M. Polyakov and A.B. Zamolodchikov, Fractal Structure of $2 D$ Quantum Gravity, Mod. Phys. Lett. A 3 (1988) 819 [InSPIRE].

[26] A.B. Zamolodchikov, On the three-point function in the minimal Liouville gravity, hep-th/0505063 [INSPIRE].

[27] A.A. Belavin and A.B. Zamolodchikov, Integrals over moduli spaces, ground ring and four-point function in minimal Liouville gravity, Theor. Math. Phys. 147 (2006) 729 [INSPIRE].

[28] M.R. Douglas, Strings in Less Than One-dimension and the Generalized KdV Hierarchies, Phys. Lett. B 238 (1990) 176 [INSPIRE].

[29] D. Gepner, Fusion rings and geometry, Commun. Math. Phys. 141 (1991) 381 [InSPIRE].

[30] M. Noumi, Expansion of the Solutions of a Gauss-Manin System at a Point of Infinity, Tokyo J. Math. 7 (1984) 1.

[31] B. Blok and A. Varchenko, Topological conformal field theories and the flat coordinates, Int. J. Mod. Phys. 7 (1992) 1467.

[32] A. Losev, 'Hodge strings' and elements of K. Saito's theory of the primitive form, hep-th/9801179 [INSPIRE].

[33] A. Belavin, D. Gepner and Y. Kononov, Flat coordinates for Saito Frobenius manifolds and String theory, arXiv:1510.06970 [INSPIRE].

[34] M. Saito, On the structure of Brieskorn lattice, Ann. Inst. Fourier (Grenoble) 39 (1989) 27. 
[35] A. Klemm, S. Theisen and M.G. Schmidt, Correlation functions for topological Landau-Ginzburg models with $c \leq 3$, Int. J. Mod. Phys. A 7 (1992) 6215 [inSPIRE].

[36] C. Li, S. Li, K. Saito and Y. Shen, Mirror symmetry for exceptional unimodular singularities, arXiv: 1405.4530 [INSPIRE].

[37] C. Li, S. Li and K. Saito, Primitive forms via polyvector fields, arXiv:1311.1659 [INSPIRE].

[38] A. Belavin and V. Belavin, On exact solution of topological CFT models based on Kazama-Suzuki cosets, J. Phys. A 49 (2016) 41LT02 [arXiv:1606.05366] [InSPIRE].

[39] V. Belavin and N. Wyllard, $N=2$ superconformal blocks and instanton partition functions, JHEP 06 (2012) 173 [arXiv: 1205.3091] [INSPIRE]. 